\title{
Effects of Upstream Component and Air Injection on Water Droplet Impingement Characteristics for Downstream Surfaces
}

\author{
Xiaobin Shen $\mathbb{D}^{1,2}$ Yundan Tan, ${ }^{1}$ Rendong Yu, ${ }^{1}$ Xiaochuan Liu, ${ }^{1}$ Guiping Lin, ${ }^{1}$ Zhiqiang Xu, ${ }^{3}$ \\ and Yuandong Guo $\mathbb{D i}^{1}$ \\ ${ }^{1}$ Laboratory of Fundamental Science on Ergonomics and Environmental Control, School of Aeronautic Science and Engineering, \\ Beihang University, Beijing 100191, China \\ ${ }^{2}$ Key Laboratory of Icing and Anti/De-Icing, China Aerodynamics Research and Development Center, Mianyang, \\ Sichuan 621000, China \\ ${ }^{3}$ Wuhan Aviation Instrument Corporation, Wuhan 430074, China
}

Correspondence should be addressed to Yuandong Guo; guoyd@buaa.edu.cn

Received 9 April 2021; Revised 19 May 2021; Accepted 12 June 2021; Published 24 June 2021

Academic Editor: Giovanni Delibra

Copyright (c) 2021 Xiaobin Shen et al. This is an open access article distributed under the Creative Commons Attribution License, which permits unrestricted use, distribution, and reproduction in any medium, provided the original work is properly cited.

\begin{abstract}
Water droplet changes its movement direction and velocity when it bypasses an aircraft component with the surrounding airflow or gets blown by air injection from the inner part. When the deflected droplet impacts on the downstream surface, its impingement characteristics would be different from those without the frontal effects. In this article, a Lagrangian method was developed to include those upstream effects on the droplet collection efficiency. Validation cases were carried out for a cylinder and an MS (1)-0317 airfoil, whereas a multielement airfoil and an engine cone with a hot air film-heating anti-icing system were computed to investigate the effects of the upstream component and air injection on the impingement characteristics of downstream surfaces. It is found that the present collection efficiencies are in good agreement with the experimental data and the simulation results obtained by the Eulerian and traditional Lagrangian methods when not affected by those upstream factors. The droplet deflections and trajectory crossings are observed clearly under the influence of the upstream component, and the Lagrangian results of downstream surfaces differ from those of the Eulerian method. In addition, due to the air injection from the inner engine cone, the peak collection efficiency on the cone surface increases with the decrease of the droplet diameter and the value even exceeds one when the droplet is small. This work is helpful for the understanding of the droplet motion and the accuracy of aircraft icing simulation.
\end{abstract}

\section{Introduction}

Aircraft icing is a great threat to flight safety and potential cause of aviation accidents and incidents, which has raised a wide concern. Normally, aircraft icing is the phenomenon that the super-cooled droplets in clouds impinge on the aircraft components and freeze on their surfaces [1]. Where and how many droplets impact on the body determine the position and extent of the ice accretion. Those are defined as droplet impingement characteristics and mainly measured by collection efficiency. To make an ice accretion analysis [2] or design an aircraft anti-icing system [3], it is essential to obtain the local collection efficiency first.
Factors that affect the super-cooled droplet motion and surface impingement characteristics include the diameter of the droplets, shape of the aircraft components, and flight status like the altitude, velocity, and attitude [4]. Various components with complex surfaces are mounted on aircrafts, and the one ahead can affect the air flow around the ones in the downstream. When water droplets bypass the upstream surface along with the airflow, their trajectories deflect or even cross due to the influence of inertial force [5]. The spatial distribution of water droplets and the distance between the adjacent droplets and other characteristics differ from the situation in the free stream, affecting the local collection efficiency on the downstream surface. For example, when the 
downstream main wing or flap of a multielement wing is obscured by the slat, no or less water droplets will hit the leading edge [6]. The upstream effects of the components in front and the air injection on the droplet impingement characteristics of downstream surfaces would be more evident on aircraft engine components. Firstly, the air flow and droplet trajectories in the S-shaped inlet duct of a jet engine would be deflected several times, which in turn affects the droplet impact on the downstream surfaces [7]. Secondly, the engine nose cone, guide vanes, and rotating blades would always be closely spaced along the engine shaft, so the droplet collection efficiencies of the downstream surfaces are inevitably influenced by the frontal components $[8,9]$. Last but not least, when hot air film-heating anti-icing systems are adopted for the engine cones and blades, the air injection from those systems can change the properties such as the airflow field and the movement direction of the water droplets, which thereby affects the downstream impact characteristics [10]. Generally, in numerical simulations of water droplet impingements, the effects of the upstream components and air injections need to be taken into account, especially for engine components.

There are mainly two methods for the droplet collection efficiency: Lagrangian method and Eulerian method. The Lagrangian method tracks the movements of water droplets in the airflow field and obtains the collection efficiency by analyzing the impingement trajectories onto the aircraft surfaces [11]. The solution process is relatively intuitive and easy for two-dimensional (2D) or geometrically simple surfaces, but for three-dimensional (3D) or complex shapes, it is difficult to determine the particle releasing positions and a large number of trajectories has to be computed [12]. The Lagrangian method was employed for the droplet collection efficiency by the NASA Glenn ice accretion code LEWICE [13]. And NASA has compared the LEWICE predictions with lots of data from droplet impingement experiments for a variety of different clean airfoils, the airfoils with simulated ice shapes, multielement wings, S-duct engine inlet, etcetera [14-16]. Good agreements were obtained for most of the tested cases, verifying the effectiveness of the Lagrangian method for various complex surfaces. As shown in the particle trajectory diagrams given in the references $[15,16]$, water droplets that fail to hit the surface were deflected in their movement directions after bypassing the airfoil boundary, especially for the simulated ice shapes, and their trajectories became denser with some crossings. Dong et al. [10] calculated the droplet impingement characteristics for an aeroengine cone with a hot air film-heating anti-icing system by the Lagrangian method. Due to the combined effect of the upstream bump and the hot air injection from the antiicing system, the water droplets were deflected before impacting on the main cone surface and a crossover of the trajectories was clearly seen. Besides, the trajectory shifts and crossings after droplets' flow over the upstream surface boundary have also been observed in other literatures [5, 17]. It is suggested that the phenomena of deflections and crossings of the droplet trajectories are objective but their effects on the downstream impingement characteristics have received little attention. The water droplet crossings were described in a NASA validation program for a 3D water droplet trajectory code using an ECS inlet geometry [18]. The trajectory crossings were believed to be caused by bad least square coefficients in certain flow field cells and resulted in irregular and incorrect water impingement patterns on the surface. Through code improvement, trajectory crossings of small-diameter droplets remained, affecting the collection efficiency distribution. It can be concluded that the upstream effects influence the droplet motion and may lead to crossing trajectories, which in turn affects the local droplet collection efficiency downstream.

The Eulerian method, on the other hand, considers the water droplet as a continuous phase and, after introducing the concept of the droplet volume fraction, obtains the distributions of the droplet volume fraction and velocity at spatial grid cells by solving the continuity and momentum equations of the droplet phase [19]. This method is a field-theoretic idea and an air-droplet two-phase flow approach, which can solve for the local collection efficiencies of all 3D surfaces at once. In the simulations of the ice accretion processes and anti-icing systems in the FENSAP-ICE software, the droplet collection efficiency is obtained by a $3 \mathrm{D}$ Eulerian method [20]. This method of FENSAP-ICE has already been utilized in the droplet calculation for complex components like 3D wings, rotorcraft, and jet engines [21, 22]. In addition, Wirogo and Srirambhatla [19] presented a Eulerian approach in FLUENT software and validated it for $2 \mathrm{D}$ and $3 \mathrm{D}$ bodies. Then, the Eulerian method was used for a 3D multielement wing and the collection efficiency was obtained on the slat, main wing, and flap, which showed the shading effect of the upstream surfaces. In the Eulerian method, droplet velocity is a single value function in each cell and the streamlines of water droplets never intersect each other. When the trajectories of water droplets cross in practice, an infinite density impulse would occur, resulting in local singularity of droplet concentration and numerical instability in the Eulerian method [12]. Stabilization terms [23], such as numerical diffusion [12] and artificial viscosity [20], should be added to eliminate the droplet oscillations and ensure convergence. Therefore, although the Eulerian method can partly track the occlusion effects of the upstream surfaces on droplet motion, it cannot capture the trajectory crossings and impingement characteristics accurately. With the enhanced region of droplet volume fraction diffused by the stabilization terms, the Eulerian collection efficiency for downstream surfaces might differ from the actual situation.

Since the Eulerian method was a relatively new approach, its results were usually compared with those obtained by the traditional Lagrangian method. It turned out that the two methods obtained consistent results for common surfaces such as cylinder and single airfoils $[12,19]$. However, when Iuliano et al. [24] performed droplet impingement prediction on a multielement airfoil with the Eulerian and Lagrangian methods, it was found that the Lagrangian limit trajectories of the water droplets deviated far away from the Eulerian droplet streamlines after bypassing a surface boundary. In addition, the collection efficiencies of the two methods agreed very well on the frontal main multielement component but differ a lot on the downstream flap surface. Those results reflected the fact that the droplet impingement characteristics 
of downstream surfaces can be affected by the upstream effects and the Eulerian and Lagrangian methods tracked those effects in different ways, leading to different results. The two methods might not be equivalents under those upstream effects.

In general, water droplets are deflected or crossed by the upstream effects such as component airflow bypassing or anti-icing hot air injection, which determines the droplet impingement characteristics on the downstream surfaces. Droplet trajectory deflections and crossings can be found in the literatures with the Lagrangian method, but their influence has received little attention, and the traditional definition of the droplet collection efficiency did not consider those conditions. In contrast, the Eulerian method is unable to capture the droplet crossings and thus cannot get an accurate pattern of the upstream effects. In this paper, we investigate the effects of the upstream component and air injection on the motion and impingement characteristics of the supercooled water droplets in clouds and establish a numerical solution to analyze the mechanism of the effects. Firstly, the droplet deflections and trajectory crossings under the effects of the upstream objects and air injection in front are described in Section 2, and a Lagrangian method with a new definition of the droplet collection efficiency is developed to consider those effects. Then, a cylinder and an MS (1)-0317 airfoil are used to validate the Lagrangian method in Sections 3.1 and 3.2, and the Lagrangian and Eulerian methods obtain almost the same results. After that, the effect of the upstream component on droplet motion is studied for a multielement airfoil, and different results appear in the two methods and are analyzed in detail. At last, an engine cone with a hot air film-heating anti-icing system is computed for the combined effects of the upstream component, and air injection and complex distributions of collection efficiency are found on the downstream cone surface with the maximum value larger than one. Since LEWICE uses the traditional Lagrangian method and FENSAP-ICE adopts only the Eulerian method for the droplet impingement characteristics, this study is useful for the better understanding of the droplet motion and the improvement of the droplet calculation under the effects of upstream components and air injections.

\section{Numerical Simulation Methods}

Since the Eulerian approach cannot capture trajectory crossings, the Lagrangian method is adopted to simulate the motion of water droplets and study the upstream effects on the impingement characteristics. In the Lagrangian framework, the movement of each particle is calculated individually to get its path line and determine whether it impinges on the aircraft surface or not. By integrating many trajectories, the collection efficiency can be obtained. Efforts have been made to reduce the droplet number to improve the efficiency of the Lagrangian method [5], for the computational costs are very expensive when a very large number of droplets are tracked for complex surfaces and 3D objects. However, when water droplets are affected by the frontal factors, it is necessary to compute lots of trajectories to capture their deflections and crossings. Therefore, the efficient Lagrangian algorithms with less tracked particles are not introduced in this work. In addition, the traditional definition of the droplet collection efficiency does not work when the droplet trajectories intersect each other and cannot express the droplet impingement characteristics under the upstream effects. Therefore, the traditional one-way coupling Lagrangian method is extended with a new definition of the droplet collection efficiency to consider the trajectory crossings. The mathematical model of the Lagrangian method is presented in Section 2.1, and the solution process and simulation steps are illustrated in Section 2.2.

2.1. Mathematical Model. The following assumptions are made for the motion of a water droplet in the airflow field $[5,12]$ : (1) the droplet remains spherical with no deformation, breaking, or splashing, (2) droplets do not have mutual influence, and neither collisions nor coalescence occur, and (3) the drag force of the surrounding air is the only one exerting on the droplet and all the others including gravity are ignored. Then, the movement of the water droplet can be determined by Newton's second law, expressed as [19].

$$
\frac{d u}{d t}=K\left(u_{a}-u\right)
$$

where $u$ is the droplet velocity vector, $t$ is the time, $u_{a}$ is the air velocity vector, and $K$ is the air-droplet exchange coefficient, given as [5]

$$
K=\frac{18 \mu_{a}}{\rho d^{2}} \frac{C_{D} \operatorname{Re}}{24},
$$

where $u_{a}$ is the air viscosity, $\rho$ is the droplet density, $d$ is the droplet diameter, and $C_{D}$ is the drag coefficient. Re is the relative Reynolds number and is calculated by

$$
\operatorname{Re}=\frac{\rho_{a}\left|u_{a}-u\right| d}{\mu_{a}}
$$

where $\rho_{a}$ is the air density.

The path line of a water droplet can be obtained by integrating the equation of the particle motion (equation (1)) through the surrounding airflow field. With all the trajectories of the droplets ending on the object surface, the collection efficiency $\beta$ can be obtained, which is defined in LEWICE as the efficiency with which any location on the object surface will collect water droplets [13]. In the Lagrangian framework, the local collection efficiency at each point on the object is usually calculated by $[5,13]$

$$
\beta=\frac{d y}{d s},
$$

where $d s$ is the surface wrap distance between the trajectories of neighbouring droplets on the object and $d y$ is the distance of the initial positions of the corresponding particles. Since $d s$ is usually larger than $d y$ due to the inertia of the droplet, 


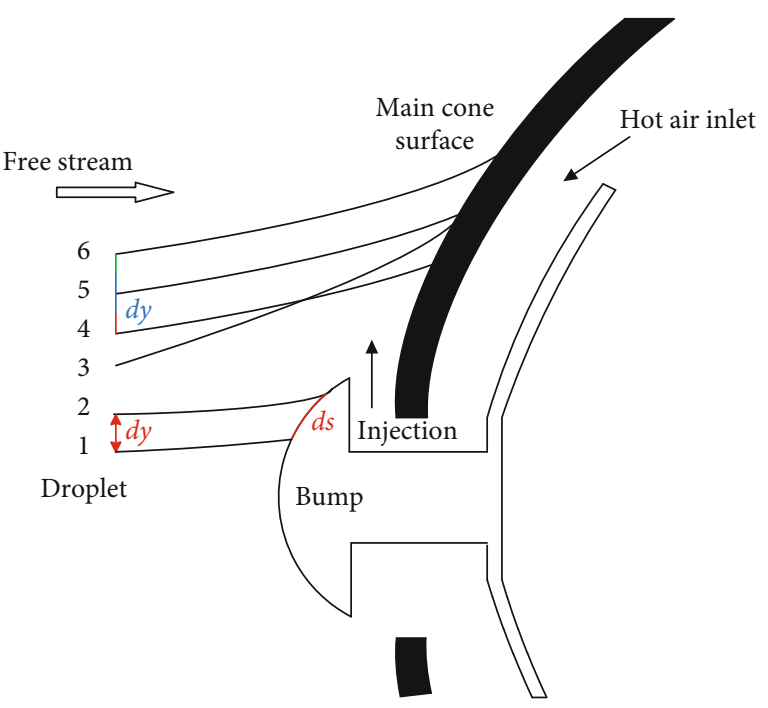

FIGURE 1: Droplet trajectories and collection efficiency under upstream effects.

collection efficiency is always below one $[8,25]$, as the case between droplet no. 1 and droplet no. 2 in Figure 1.

However, when droplet motion is deflected by upstream effects, it is possible that $d y$ becomes larger than $d s$, resulting in a collection efficiency bigger than one. Figure 1 presents a sketch map of an engine cone with a hot air anti-icing system, which is similar to that described in the reference [10]. When droplets bypass the upstream bump of the cone, their trajectories change directions and then end on the downstream main cone surface, as shown in Figure 1. In addition, due to the air injection from the anti-icing system in front of the main cone, droplets would move away from the jet site and the closer to the hot air outlet, the greater the change of the movement direction. As a result of those combined effects, $d s$ between droplet no. 4 and droplet no. 5 is smaller than $d y$ and the collection efficiency bigger than one is obtained there. Moreover, trajectory crossings would happen before droplet impinging on the object. Therefore, two droplets could impact on the same location (see droplet no. 3 and no. 5 in Figure 1), causing an infinite value of $\beta$. Furthermore, the value of $d s$ between the $3^{\text {rd }}$ and $4^{\text {th }}$ droplets is even negative. It can be seen that the local collection efficiency under the upstream effects could not be obtained by equation (4) anymore.

In this work, the Lagrangian method is extended to adapt to these conditions in consideration of upstream effects. The mass flux-based collection efficiency mentioned in reference [8] is introduced, which is the ratio of the local droplet mass flux at the object surface to its value at the far field inlet. When the initial distances between the adjacent releasing points are the same and all equal to $d y$, the water droplet can be considered as a microelement with a width of $d y$ instead of just a massless point (see droplet no. 5 in Figure 1), and then, the mass flux of a droplet in $2 \mathrm{D}$ conditions would be

$$
m=\mathrm{LWC} \cdot u_{\infty} \cdot d y
$$

where LWC is the liquid water content (grams of water contained in a cubic meter of air) and $u_{\infty}$ is the freestream velocity. When a droplet trajectory ends in a control volume (CV) on the surface, the mass flux of the droplet is absorbed by the $\mathrm{CV}$. Hence, the total mass flux of the impingement droplets in the $i^{\text {th }} \mathrm{CV}$ can be calculated according to the total number $N$ of the droplet trajectories ending in the CV and its local droplet collection efficiency on the surface can be obtained by

$$
\beta_{i}=\frac{\mathrm{LWC} \cdot u_{\infty} \cdot d y \cdot N}{\mathrm{LWC} \cdot u_{\infty} \cdot \Delta s_{i}}=\frac{N \cdot d y}{\Delta s_{i}},
$$

where $\Delta s_{i}$ is the curve length of the $i^{\text {th }} \mathrm{CV}$. According to equation (6), the trajectory crossings and the condition of the same impingement location can be considered in the extended Lagrangian method. The mass flux-based collection efficiency, which is similar to the definition in the Eulerian method, is the average of the traditional results in a control volume.

2.2. Solution Procedure. The droplet impingement simulation based on the extended Lagrangian method is carried out in the commercial CFD software Ansys FLUENT-19.1 with its user-defined functions (UDFs) [26]. Firstly, the steady airflow field is obtained by solving the Reynolds averaged Navier-Stokes equations (RANS) with the finite volume solver of FLUENT. Droplet motion is insensitive to the airflow in the boundary layer, and even the inviscid flow fields work well for the droplet impingement characteristics [12]. In this work, the one-equation Spalart-Allmaras turbulence model is used to catch the upstream effects on the air flow. However, the dispersion of droplets due to turbulence is not considered. The SIMPLE (semi-implicit method for pressure-linked equations) algorithm is employed with the discretization of the second-order upwind scheme for the CFD calculation. Since the LWC and droplet diameter in the cloud is small, the aerodynamic effects of droplets on the airflow field are negligible [27]. Then, the droplet motion is decoupled from the airflow calculation (one-way coupling) and solved in a freezing airflow field with a fourth-order Runge-Kutta scheme. The solution of a droplet path line starts at a released location far away from the object surface where the air velocity is the same with the freestream value and ends with a trap boundary condition when it intersects the object surface or reaches the downstream far field boundary. The tracked particles are more than $10^{5}$ to guarantee the independence of the number of the droplets injected in the Lagrangian method. At last, UDFs are used to integrate the trajectories ending in each CV on the object surfaces and evaluate the local collection efficiency by equation (6).

\section{Results and Discussions}

To verify the present Lagrangian method, the known droplet validation cases of a cylinder and an MS (1)-0317 airfoil are used by comparing the collection efficiency and impingement limits to the experimental data and the simulation results from the literatures. Then, a multielement airfoil 
TABLE 1: The Langmuir-D distribution for MVD $=16 \mu \mathrm{m}$.

\begin{tabular}{lcc}
\hline No. & $w_{j}(\%)$ & $d(\mu \mathrm{m})$ \\
\hline 1 & 5 & 5.0 \\
2 & 10 & 8.3 \\
3 & 20 & 11.4 \\
4 & 30 & 16.0 \\
5 & 20 & 21.9 \\
6 & 10 & 27.8 \\
7 & 5 & 35.5 \\
\hline
\end{tabular}

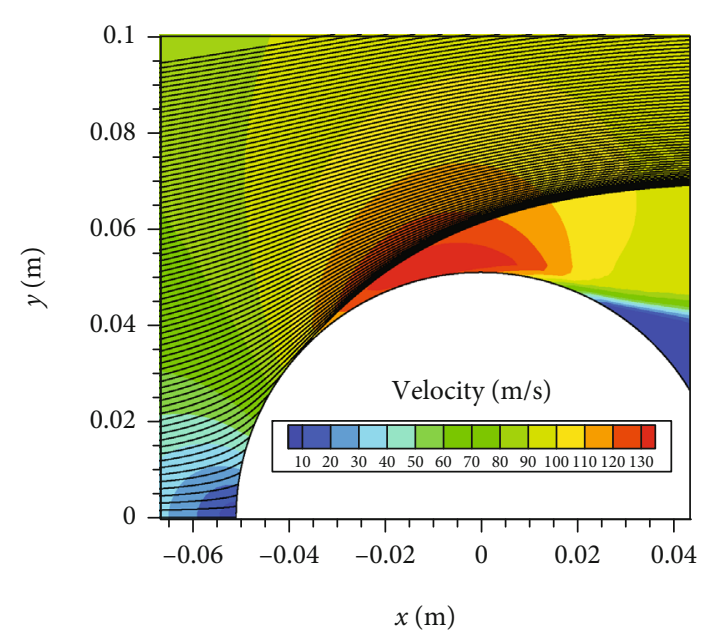

FIGURE 2: Lagrangian droplet trajectories on contour of air velocity around the cylinder at the droplet diameter of $16 \mu \mathrm{m}$.

and a section of an engine cone are selected to study the upstream effects of the frontal object and air injection.

3.1. Cylinder. The first validation case is a circular cylinder with a diameter of $10.16 \mathrm{~cm}$ in reference [12]. The freestream velocity is $80 \mathrm{~m} / \mathrm{s}$, and the density of air is set to be 1.097 $\mathrm{kg} / \mathrm{m}^{3}$ with an ambient pressure of $89867 \mathrm{~Pa}$. Seven different droplet sizes with a median volumetric diameter (MVD) of $16 \mu \mathrm{m}$, which satisfy the Langmuir-D distribution, are computed separately for the droplet impingement characteristics, as listed in Table 1 . The composite collection efficiency in a distribution of a droplet size can be obtained by [12]

$$
\beta=\sum_{j=0}^{M} w_{j} \beta_{j},
$$

where $M$ is the total number of the droplet sizes, $w_{j}$ is the percentage of the $j^{\text {th }}$ droplet group, and $\beta_{j}$ is the corresponding collection efficiency.

Figure 2 shows the contour of air velocity around the cylinder, along with the droplet trajectories obtained by the Lagrangian method. In front, far away from the cylinder, water droplets keep their motion at the free air velocity. When they are close to the cylinder, their movement directions change because of the air flow around the surface and the trajectories deviate from the air streamlines due to the

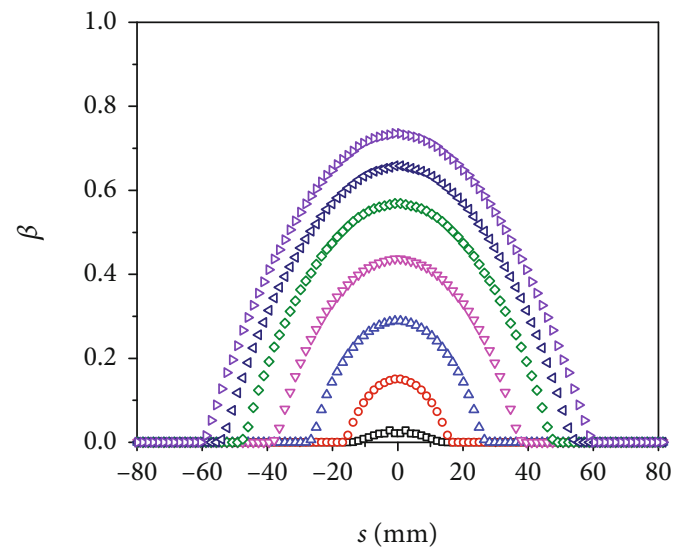
$d=5 \mu \mathrm{m}$
$\diamond d=21.9 \mu \mathrm{m}$
○ $d=8.3 \mu \mathrm{m}$
$\triangleleft d=27.8 \mu \mathrm{m}$
$\Delta d=11.4 \mu \mathrm{m}$
$\triangleright d=35.5 \mu \mathrm{m}$
$\nabla d=16 \mu \mathrm{m}$

FIgURE 3: Collection efficiency of the cylinder for various droplet sizes at the MVD of $16 \mu \mathrm{m}$ in Langmuir-D distribution.

inertia. Parts of droplets impinge on the leading edge of the cylinder, and the droplet impingement limits are located at the points where the trajectories are tangent to the cylinder surface. For the droplets failing to impact on the cylinder, the one relatively closer to the cylinder is affected more sharply by the airflow change, leading to a stronger deflection, while the airflow's influence is rather limited on the droplets away from the surface. Due to this reason, a concentrated zone of the droplet trajectories is formed near the rear of the cylinder. Even more, trajectory overlaps and crossings are found in some certain areas.

The collection efficiencies of the seven droplet sizes in the Langmuir-D distribution are presented in Figure 3. The local collection efficiency distributes symmetrically on the cylinder surface, summits at the stagnation point, and decreases alongside both the pressure and suction surfaces until it becomes zero at the impingement limits. It can be seen that the droplet diameter has a strong connection with the impingement characteristics. When the droplet size is larger, which means bigger inertia and larger deviation from the air streamlines, it would result in a wider impingement region and larger peak collection efficiency.

Comparison of collection efficiency between the composite value with Langmuir-D distribution and the experimental data is presented in Figure 4, where the shaded region is the range of the experimental data. It can be seen that the peak local collection efficiency obtained in this work is a little smaller than that obtained by the Eulerian method [12] but the similar impingement regions are attained in both methods. In addition, the simulation result matches well with the experimental data, which validates the present Lagrangian method.

3.2. MS (1)-0317 Airfoil. An MS (1)-0317 airfoil with a chord length of $0.9144 \mathrm{~m}$ is taken from the Papadakis' droplet impingement experiments [16] as the second validation case 


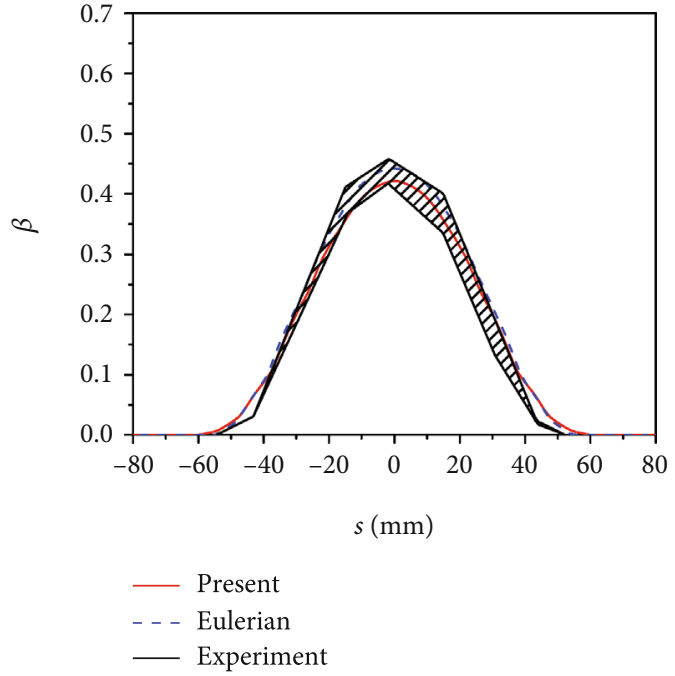

Figure 4: Comparison of collection efficiency with experimental and Eulerian results for the cylinder.

TABLE 2: The 10-bin droplet size distribution for MVD $=21 \mu \mathrm{m}$.

\begin{tabular}{lcc}
\hline Bin no. & $w_{i}(\%)$ & $d(\mu \mathrm{m})$ \\
\hline 1 & 5 & 4.040659 \\
2 & 10 & 9.67207 \\
3 & 20 & 14.24772 \\
4 & 30 & 20.9438 \\
5 & 20 & 28.15316 \\
6 & 10 & 45.23621 \\
7 & 3 & 70.07175 \\
8 & 1 & 88.85927 \\
9 & 0.5 & 103.4068 \\
10 & 0.5 & 163.9674 \\
\hline
\end{tabular}

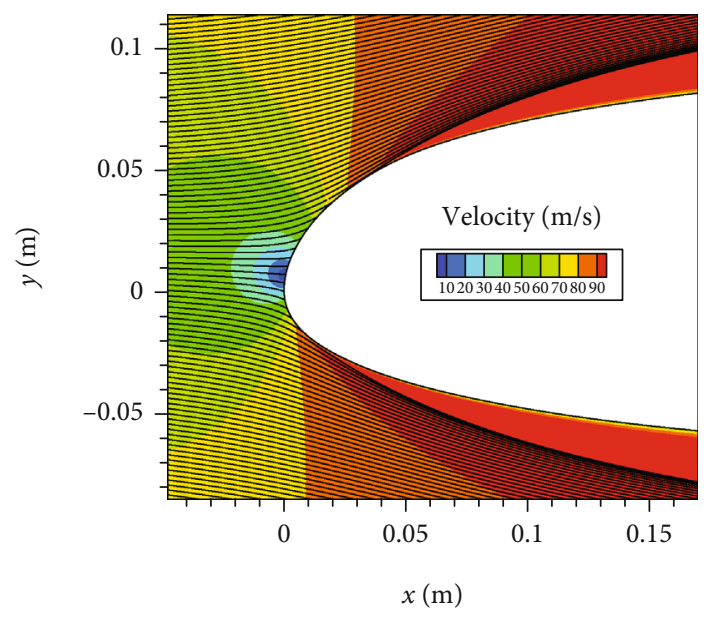

FIGURE 5: Lagrangian droplet trajectories on contour of air velocity around the MS (1)-0317 airfoil at the droplet diameter of $20.9 \mu \mathrm{m}$.

for the present Lagrangian method. The test condition is at the freestream velocity of $78.23 \mathrm{~m} / \mathrm{s}$ with the MVD of 21 $\mu \mathrm{m}$. The droplet size distribution was measured in the exper-

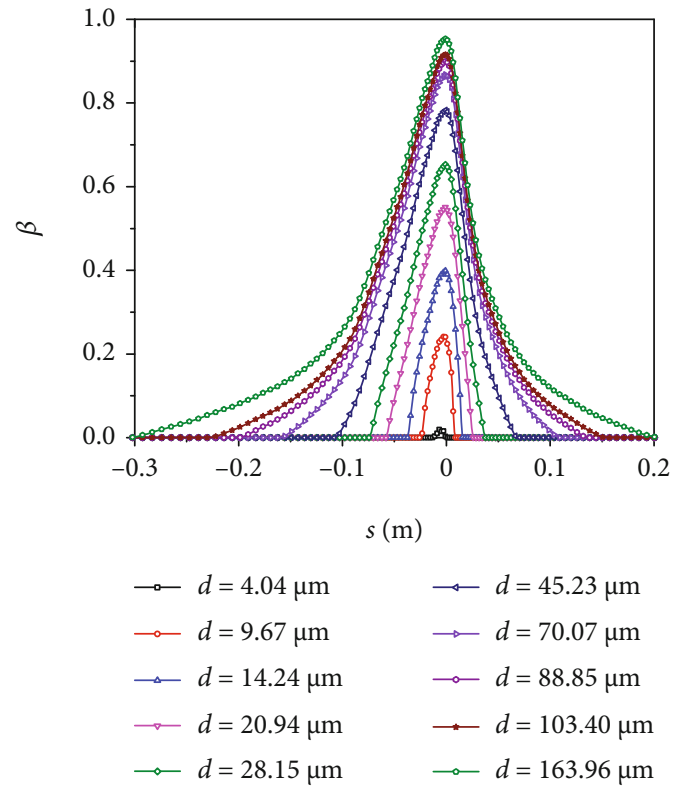

FIGURE 6: Collection efficiency of the MS (1)-0317 airfoil for various droplet sizes at the MVD of $21 \mu \mathrm{m}$ in the 10-bin distribution.

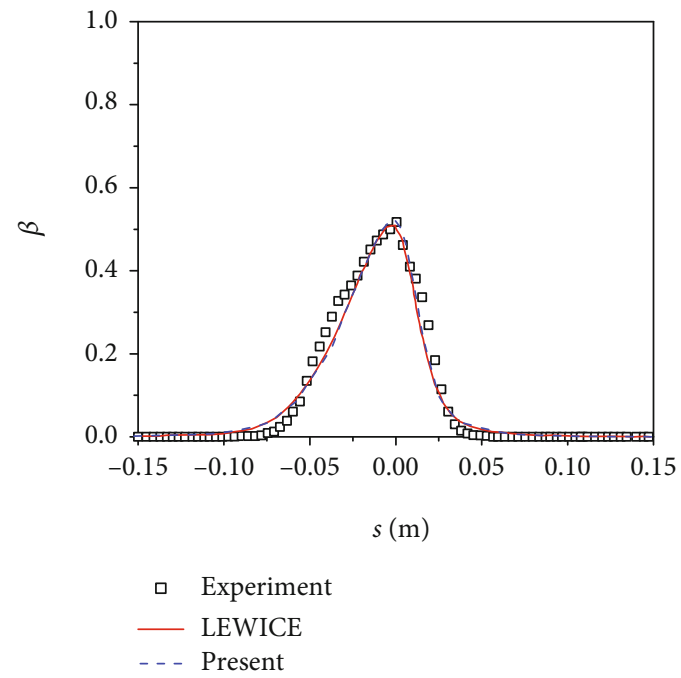

FIgURE 7: Comparison of collection efficiency with experimental and LEWICE results for the MS (1)-0317 airfoil.

iment, and a 10-bin discrete droplet size distribution was generated as an approximation for the droplet impingement characteristics. The 10-bin droplet size distribution of the MVD is listed in Table 2, and the composite collection efficiency is also computed by equation (7). The test was carried out at an angle of attack (AOA) of $0^{\circ}$ in the Goodrich Icing Wind Tunnel. However, the AOA used in the LEWICE analysis and in this work is set to be $-1.85^{\circ}$ to match the experimental pressure distribution on the airfoil surface, since the airflow field around the MS (1)-0317 airfoil was affected by the narrow tunnel walls [16]. In addition, the inlet pressure is $95292 \mathrm{~Pa}$ and the air density is $1.213 \mathrm{~kg} / \mathrm{m}^{3}$.

The Lagrangian droplet trajectories and the air velocity distribution around the MS (1)-0317 airfoil are shown in 


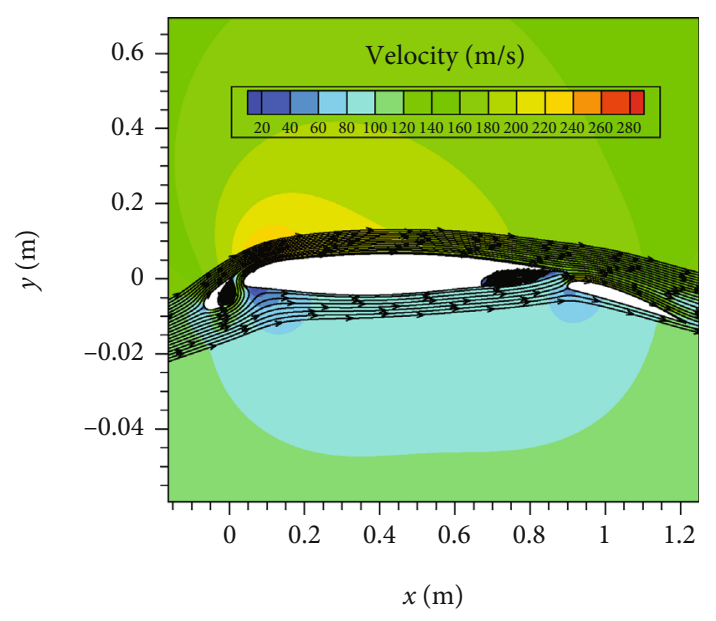

(a) Overall view

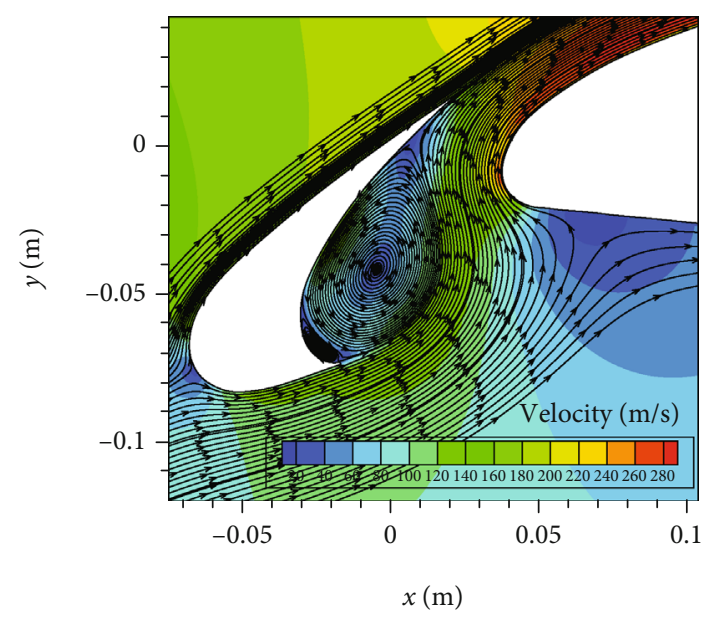

(b) Results around the slat and main wing

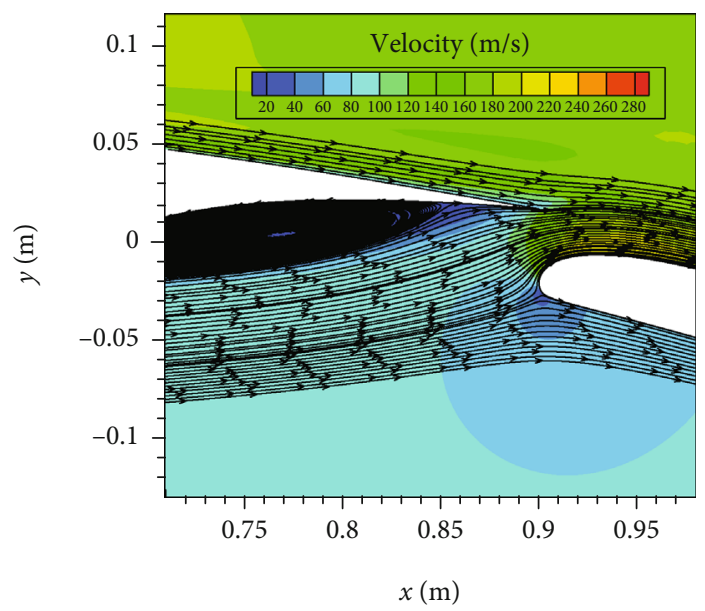

(c) Results around the flap

FIGURE 8: Contour of air velocity and the streamlines around the multielement airfoil.

Figure 5. Similar to what happened to the cylinder, the droplet trajectories do not deviate from the airflow direction until they reach the vicinity of the airfoil's leading edge. Part of the droplets impacts on the airfoil. Due to the negative angle of attack, water droplets are more likely to hit the suction side of the airfoil surface. When passing by the airfoil, the droplets closer to the surface deviate from the streamline more obviously in contrary to the further ones. Near the airfoil surface, a concentrated zone of the path lines is also observed with the overlaps and crossings of the droplet trajectories, also alike to the situation in the cylinder case. These phenomena can also be found in references $[5,26]$. Since the trajectory overlapping and crossing regions are located out of the impingement limits, they are considered to have no effects on the droplet collection efficiency and have not attracted much attention.

The collection efficiency distributions for the 10-bin droplet size are illustrated in Figure 6, where the surface distance $s=0$ means the stagnation point and the value on the upper surface is positive. All the curves reach their maxima at the stagnation point on the suction surface and decline to zero as moving backwards. The upper wing surface witnesses a slower descend in local collection efficiency and has a broader impingement region due to the negative angle of attack. With the increase of the droplet diameter, the peak collection efficiency escalates, which fits the result from the previous cylinder case as well.

The overall collection efficiency is compared with the numerical result obtained by LEWICE and the experimental data, as shown in Figure 7. It can be seen that the match between the results of the present method and LEWICE is very good, indicating that the extension to the Lagrangian method with the mass flux-based collection efficiency is effective and feasible. In addition, the numerical results are both in good agreement with the experimental data, which validates the Lagrangian approach.

3.3. Multielement Airfoil. A three-element airfoil is selected from reference [28] to study the effects of frontal surfaces on the droplet trajectories and the impingement characteristics of downstream surfaces. Numerical simulation is carried out at a freestream Mach number of 0.4 with an AOA of $4^{\circ}$. The inlet pressure is $80 \mathrm{kPa}$ with the ambient temperature of $260 \mathrm{~K}$. A droplet diameter of $20 \mu \mathrm{m}$ is used for the droplet motion computation. It can be seen from the contour of the air velocity and the streamlines around the multielement airfoil in Figure 8 that a complex flow field is formed with 


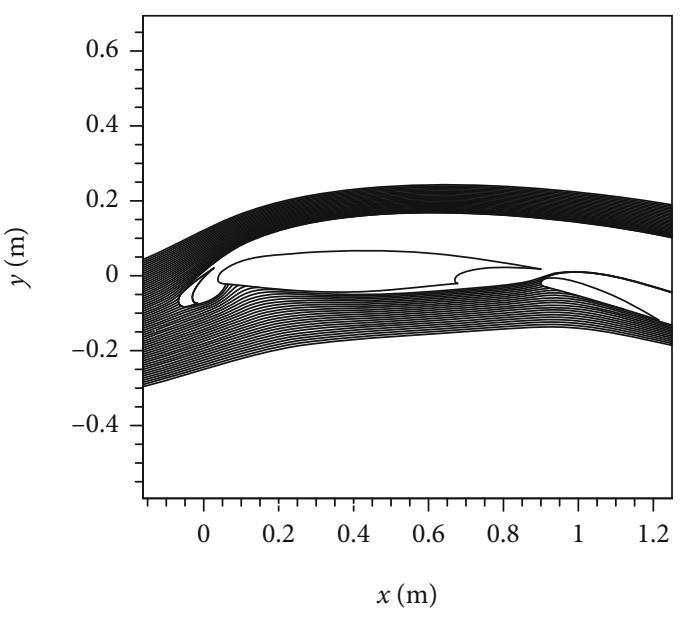

(a) Overall view

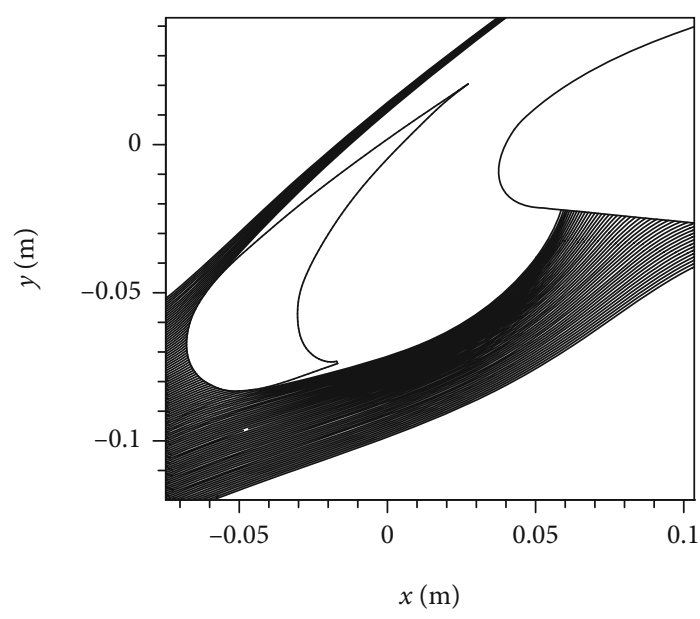

(b) Results around the slat and main wing

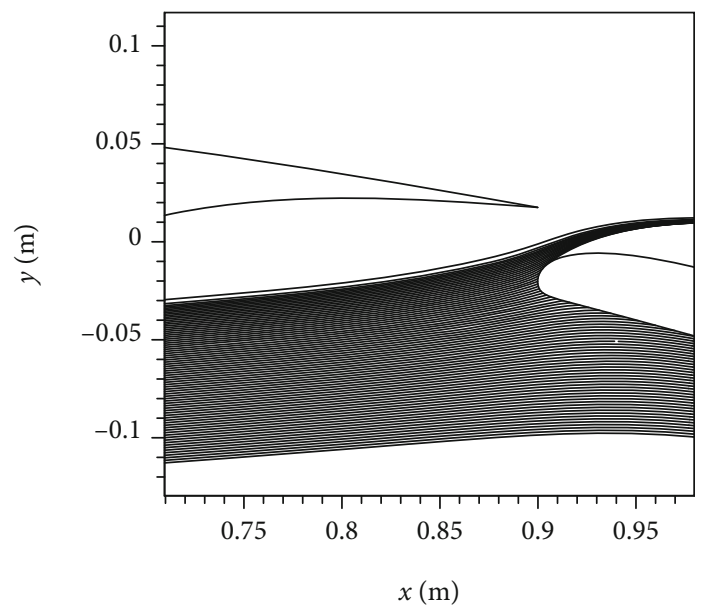

(c) Results around the flap

FIgURE 9: Lagrangian droplet trajectories around the multi-element airfoil at the droplet diameter of $20 \mu \mathrm{m}$.

vortexes, since the tailing edges of the slat and main wing do not transient smoothly with air streamlines and have large windward areas. The wake of the forward bodies interacts with the airflow around the multielement airfoil, which distorts the droplet trajectories and affects the local collection efficiency on the downstream surfaces. Especially in the area between the slat and the main wing, the slat is located lower than the main wing and they are so close to each other. At a positive angle of attack, the air has to bypass the lower slat surface, flows through the space between the slat and the main wing, and then reaches the leading edge of the main wing. The airflow is divided into two streams, which flow through the pressure and suction surfaces of the main wing, as shown in Figure 8. It makes the stagnation point appear at a lower location and creates an even more complex airflow in front of the main wing.

From the trajectories demonstrated in Figure 9, water droplets impinge on the slat and the pressure surface of the main wing and the front part of the flap. In the rear area of the slat, the trajectories missing the upstream elements form a concentrated zone, which means that the distance between the adjacent trajectories is smaller than where they start. When droplets bypass the pressure surface of the slat, they continue their trajectories to the region with a complex vortex flow between the slat and the main wing, and change their movement directions again. Some of the droplets finally impinge on the pressure surface of the main wing with an irregular distribution of the impact points. Due to droplets' inertia, none of them moves to the upper wing surface with the airflow. In the same way like the slat, a concentrated zone of the droplet trajectories is formed below the main wing. However, since the main wing has a much larger area and longer chord with a smoother profile, the distance between neighbouring trajectories do not have apparent changes before droplets arrive on the flap. In addition, the flap is located behind and below the main wing, and distant from it, so the airflow around the flap is slightly affected by the main wing or the frontal slat, leading to a smoother distribution of the impingement positions.

The collection efficiencies of the slat, main wing, and flap are compared with those obtained by the Eulerian method [28], as shown in Figure 10. It is found that the present method could successfully capture the droplet impingement regions and characteristics for the multielement airfoil. Since there is no effect of the upstream surface on the slat, the local collection efficiency there coincides very well with that 


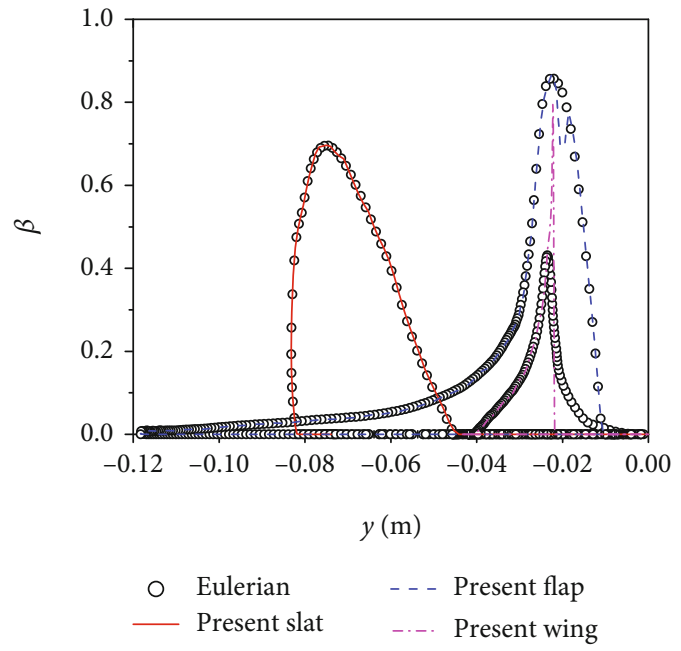

Figure 10: Comparison of collection efficiency with the Eulerian result for the multielement airfoil at the droplet diameter of $20 \mu \mathrm{m}$.

of the Eulerian method, which also validates the developed Lagrangian method. A great difference is found on the main wing surface. The present collection efficiency has a larger value, but a smaller range than the Eulerian result in the upper region. The phenomena of the same collection efficiency on the frontal surface and different results on the downstream component were also observed in the simulations with the two methods in reference [24]. This might be attributed to the different ways of dealing with droplet motion after being deflected by the upstream flow. In the Lagrangian method, droplets' trajectories are tracked one by one in the airflow and this approach works very well even when the droplet motion is strongly affected by the complex wake flow as shown in Figure 9. On the other hand, droplet velocity is a single value function in the Eulerian method [12]. When the droplet motion is deflected, two droplet streams might gather to a single direction, causing an infinite droplet volume fraction and local singularity of droplet concentration. Numerical diffusion is added in the continuity equation of the droplet phase to smooth out the local singularity in the Eulerian method [28]. When the droplet concentration due to the wake flow is diffused in front of the main wing, a smoother distribution of the collection efficiency is obtained on the wing surface.

For the same reason, the divergence in the local collection efficiency on the flap can be understandable. After the water droplets flow by the main wing, the concentrated zone is formed. The droplet concentration in this region is diffused in the Eulerian method, while the Lagrangian method captures it very well under the condition that massive droplets are tracked. Since the main wing has a larger area, the air streamlines transit slowly below the pressure surface and result in a relatively sparse distribution of the droplets. Besides, the long distance and relative position between the flap and the wing further limit the influence of the upstream on the flap. Therefore, these two methods obtain different collection efficiencies only at the frontal area of the flap and the Lagrangian result is more irregular.

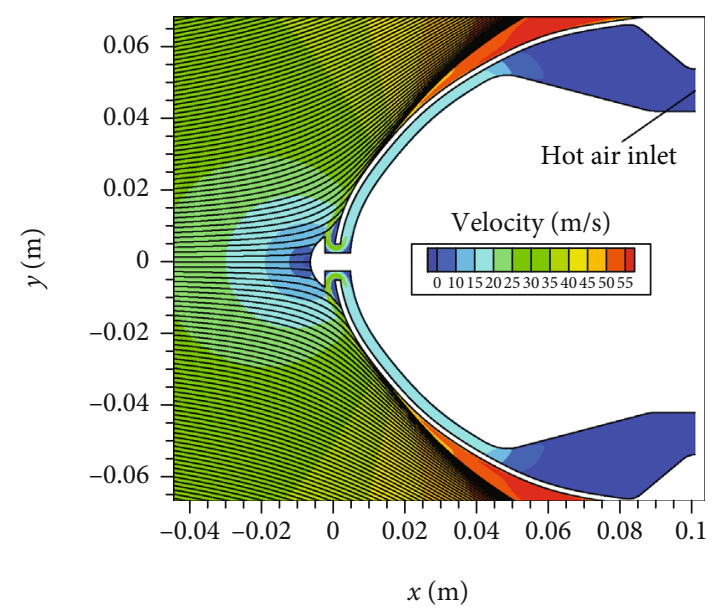

FIgURE 11: Lagrangian droplet trajectories on the contour of air velocity around the cone section at the droplet diameter of $20 \mu \mathrm{m}$.

3.4. Section of an Engine Cone. As described in Section 1, the hot air film-heating anti-icing system can be used in aeroengine cones [10]. A section of this engine cone is investigated for the effects of the upstream surface and air injection on the droplet trajectories and the impingement characteristics of the downstream surface. Since the airflow and local collection efficiency in the axisymmetric coordinate need special treatment [10], a simple $2 \mathrm{D}$ section model of the engine cone is tested, as shown in Figure 11. In addition, the temperature difference between the free stream and the anti-icing hot air is ignored to focus on the effects of the injection air flow. The freestream velocity is $40 \mathrm{~m} / \mathrm{s}$, and the ambient pressure is $1 \mathrm{~atm}$ with a temperature of $300 \mathrm{~K}$. The mass flow rate of the hot air is $0.0575 \mathrm{~kg} / \mathrm{s}$. The droplet diameters of $18 \mu \mathrm{m}, 20 \mu \mathrm{m}$, and $24 \mu \mathrm{m}$ are used in the simulation.

The air velocity field around the engine cone is shown in Figure 11, along with the Lagrangian droplet trajectories at a diameter of $20 \mu \mathrm{m}$. The freestream air flows to the frontal bump of the engine cone and then moves downstream. With the hot air jet from the outlet of the anti-icing system, the mixed air flows backward along the main cone surface. Meanwhile, the motion of the water droplet is affected both by the cone surfaces and the hot air injection, and the droplets impinge on both the bump and the downstream cone surfaces. Since the effects of the bump surface and the air injection are mainly on the downstream droplet motion, attention is fixed on the droplet impingement characteristics of the main cone surface.

The droplet trajectories near the hot air jet site around the engine cone are shown in detail in Figure 12. It can be seen that water droplets change their movement directions in front of the main cone mainly due to the hot air injection. When the droplets are at a diameter of $24 \mu \mathrm{m}$, the deflection of water droplets from their original movement trend is relatively small due to the large inertia, as shown in Figure 12(a). The distance between neighbouring trajectories becomes smaller before impact on the main cone, but no droplet crossings are observed with the sparse path lines. When the droplets' diameter is $20 \mu \mathrm{m}$, more obvious deflection is found in 


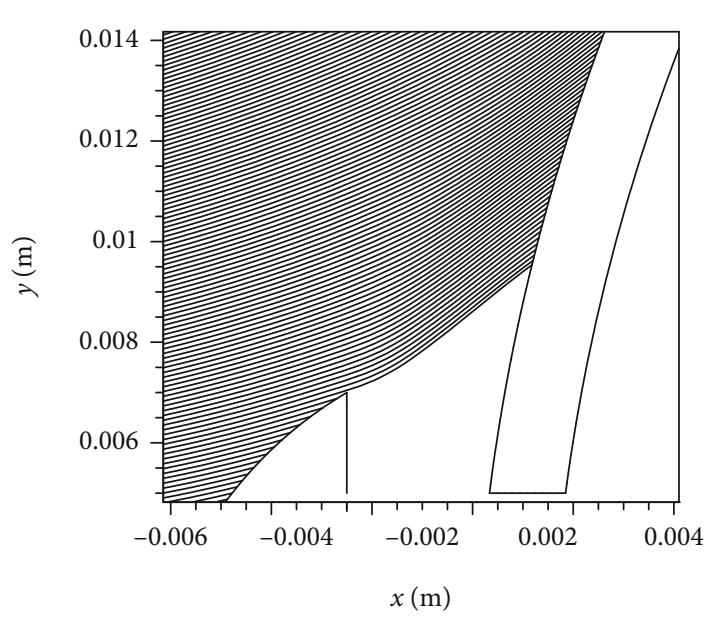

(a) Trajectories at the droplet diameter of $24 \mu \mathrm{m}$

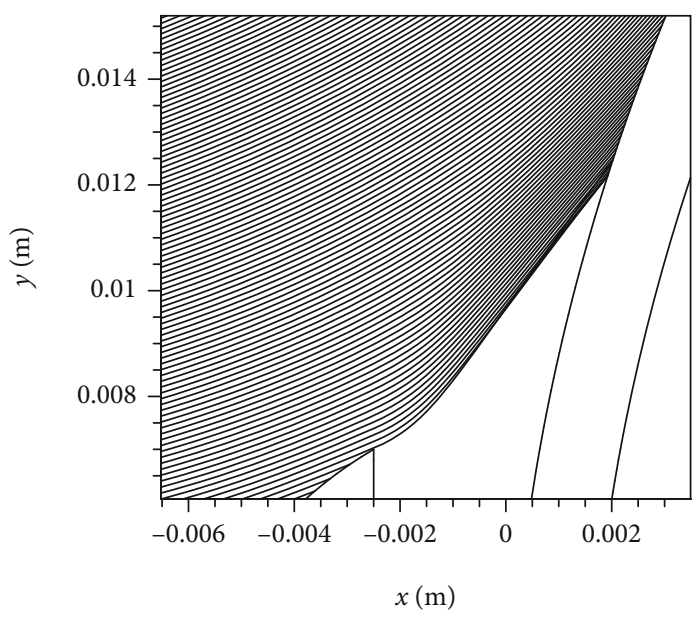

(b) Trajectories at the droplet diameter of $20 \mu \mathrm{m}$

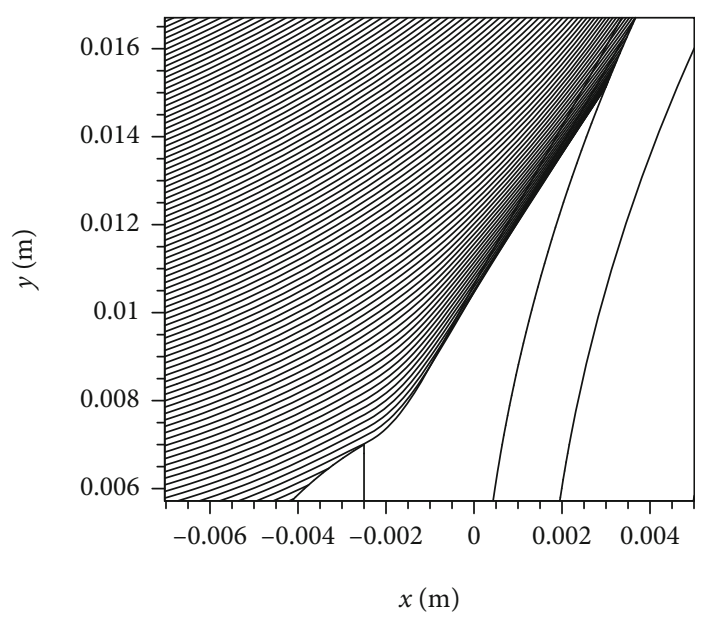

(c) Trajectories at the droplet diameter of $18 \mu \mathrm{m}$

FIGURE 12: Droplet trajectories for the engine cone section at various droplet diameters.

the trajectories and the impingement region moves further away from the symmetry axis. In addition, the droplets closer to the hot air jet site receive larger impact from the jet air flow, leading to a greater change in the direction of motion. Therefore, water droplets move close to each other and a clear illustration of trajectory crossings can be found in Figure 12(b). Similar phenomena have been observed [10], but no description or impact analysis was made. From the results at the droplet diameter of $18 \mu \mathrm{m}$ in Figure 12(c), an even more strong total deviation of the trajectories is observed and the droplet impingement region moves further downstream. Moreover, the crossing phenomenon of the droplet trajectories is more obvious and extensive.

Figure 13 lists the local collection efficiencies on the main cone at different droplet diameters. Under the upstream effects of the bump and air injection, the impingement positions concentrate close to the frontal part of the cone surface, leading to a large collection efficiency there and a rapid decrease when it moves backwards. When the diameter is larger, the droplets are more likely to impinge on the surface and the larger impingement region is obtained, which is the

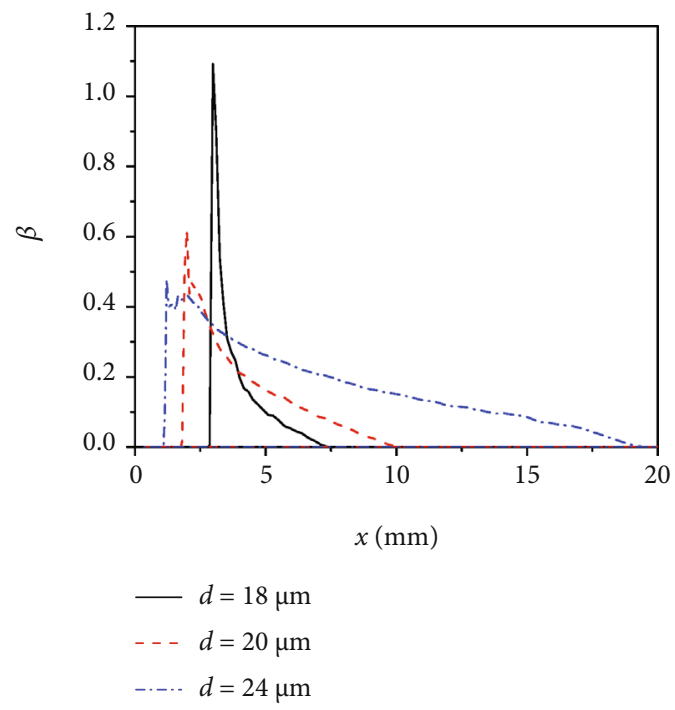

Figure 13: Collection efficiency for the engine cone section at various droplet diameters. 
same to the previous observation. However, different from the usual situation, the peak collection efficiency increases with the decrease of the droplet diameter and the local collection efficiency even exceeds one when the droplet diameter is $18 \mu \mathrm{m}$. This is because smaller droplets are easier to be deflected by the jet flow and trajectory crossings are more likely to happen with more droplets. The water would impact on a relatively small region, leading to the increase of the collection efficiency. When the number of the trajectories ending in a CV is more than that of the droplet releasing points corresponding to the $\mathrm{CV}$, meaning that the mass flux of the droplets in the $\mathrm{CV}$ is larger than the freestream value, the local collection efficiency of the $\mathrm{CV}$ is greater than one.

\section{Conclusion}

The Lagrangian method is extended with the mass flux-based collection efficiency to consider the droplet deflections and trajectory crossings under the effects of the upstream objects and air injection in front. Test cases are performed for different 2D surfaces, namely, a cylinder, an MS (1)-0317 airfoil, a multielement airfoil, and an engine cone with a hot air filmheating anti-icing system. Results of the droplet motion and local collection efficiency are investigated, and the main conclusions are as follows:

(1) The present collection efficiencies of the cylinder and MS (1)-0317 airfoil match well with the experimental data and those obtained by the Eulerian and traditional Lagrangian methods, verifying the extended method for the droplet impingement characteristics

(2) When water droplets are affected by air injection or bypass an upstream object, their motions are deflected and trajectory overlaps and crossings are observed. When the downstream component is in the affected area, its collection efficiency is influenced

(3) The collection efficiencies obtained by the Eulerian and Lagrangian methods are consistent for the surfaces without upstream effects, but the results deviate after water droplets are deflected. The two methods are not equivalents. The Lagrangian method captures the droplet trajectory deflections and crossings very well and achieves irregular impingement distribution, while the Eulerian method obtains smoother collection efficiency due to its numerical diffusion for the droplet concentration

(4) Air injection of the engine cone would affect the droplet motion, and the deflection of the droplet trajectory from its initial trend is more obvious when the diameter is smaller. At small droplet diameters, trajectory crossings are found in front of the cone surface. The peak collection efficiency increases with the decrease of the droplet diameter, and the value even exceeds one when the droplet diameter is $18 \mu \mathrm{m}$

\section{Data Availability}

The data used to support the findings of this study are available from the corresponding author upon request.

\section{Conflicts of Interest}

The authors declare that there is no conflict of interest.

\section{Acknowledgments}

This work was supported by the National Natural Science Foundation of China (no. 51806008), the National Numerical Wind Tunnel Project (no. NNW2019ZT2-A07), and the Open Fund of Key Laboratory of Icing and Anti/De-icing (no. IADL 20200307).

\section{References}

[1] Y. Cao, W. Tan, and Z. Wu, "Aircraft icing: an ongoing threat to aviation safety," Aerospace Science and Technology, vol. 75, pp. 353-385, 2018.

[2] X. Shen, G. Lin, J. Yu, C. du, and X. Bu, "Three-dimensional numerical simulation of ice accretion at the engine inlet," Journal of Aircraft, vol. 50, no. 2, pp. 635-642, 2013.

[3] J. Yu, L. Peng, X. Bu, X. Shen, G. Lin, and L. Bai, "Experimental investigation and correlation development of jet impingement heat transfer with two rows of aligned jet holes on an internal surface of a wing leading edge," Chinese Journal of Aeronautics, vol. 31, no. 10, pp. 1962-1972, 2018.

[4] A. Heinrich, R. Ross, and G. Zumwalt, Aircraft Icing Handbook. Volume 1, Atlantic City International Airport, NJ 08405: Department of Transportation, Federal Aviation Administration Technical Center, DOT/FAA/CT-88/8-1, 1991.

[5] L. Xie, P. Li, H. Chen, and H. Liu, "Robust and efficient prediction of the collection efficiency in icing accretion simulation for 3D complex geometries using the Lagrangian approach I: an adaptive interpolation method based on the restricted radial basis functions," International Journal of Heat and Mass Transfer, vol. 150, p. 119290, 2020.

[6] C. Yu, P. Ke, G. Yu, and C. Yang, "Investigation of water impingement on a multi-element high-lift airfoil by Lagrangian and Eulerian approach," Propulsion and Power Research, vol. 4, no. 3, pp. 161-168, 2015.

[7] K. Al-Khalil, R. Hitzigrath, O. Philippi, and C. Bidwell, "Icing analysis and test of a business jet engine inlet duct," in 38th Aerospace Sciences Meeting \& Exhibit, Reno, NV, 2000AIAA2000-1040.

[8] A. Hamed, K. Das, and D. Basu, "Numerical simulations of ice droplet trajectories and collection efficiency on aero-engine rotating machinery," in 43rd AIAA Aerospace Sciences Meeting \& Exhibit, Reno, NV, 2005AIAA-2005-1248.

[9] C. Bidwell, "Ice particle transport analysis with phase change for the E3 turbofan engine using LEWICE3D Version 3.2," in 4th AIAA Atmospheric and Space Environments Conference, New Orleans, Louisiana, 2012AIAA-2012-3037.

[10] W. Dong, J. Zhu, M. Zheng, and Y. Chen, "Thermal analysis and testing of nonrotating cone with hot-air anti-icing system," Journal of Propulsion and Power, vol. 31, no. 3, pp. 896-903, 2015. 
[11] C. Wang, S. Chang, and H. Wu, "Lagrangian approach for simulating supercooled large droplets' impingement effect," Journal of Aircraft, vol. 52, no. 2, pp. 524-537, 2015.

[12] X.-L. Tong and E. A. Luke, "Eulerian simulations of icing collection efficiency using a singularity diffusion model," in $43 \mathrm{rd}$ AIAA Aerospace Sciences Meeting and Exhibit, Reno, Nevada, 2005AIAA-2005-1246.

[13] W. B. Wright, "User Manual for the NASA Glenn Ice Accretion Code LEWICE Version 2.2.2," NASA Report No. NASA-CR2002-211793, National Aeronautics and Space Administration, Glenn Research Center, 2002.

[14] M. Papadakis, K. E. Hung, G. T. Vu, H. W. Yeong, and C. S. Bidwell, Experimental Investigation of Water Droplet Impingement on Airfoils, Finite Wings, and an s-Duct Engine Inlet, 2002, NASA Report no. NASA/TM-2002-211700.

[15] M. Papadakis, A. Rachman, S. C. Wong et al., Water Droplet Impingement on Simulated Glaze, Mixed, and Rime Ice Accretions, 2007, NASA Report no. NASA/TM-2007-213961.

[16] M. Papadakis, S. C. Wong, A. Rachman, K. E. Hung, G. T. Vu, and C. S. Bidwell, Large and Small Droplet Impingement Data on Airfoils and Two Simulated Ice Shapes, 2007, NASA Report no. NASA/TM-2007-213959.

[17] T. C. S. Rendall and C. B. Allen, "Finite volume droplet trajectories for icing simulation," in 28th AIAA Applied Aerodynamics Conference, Chicago, Illinois, 2010.

[18] M. D. Breer and M. P. Goodman, Three-Dimensional Water Droplet Trajectory Code Validation using an ECS Inlet Geometry, 1993, NASA Contractor Report no. 191097.

[19] S. Wirogo and S. Srirambhatla, "An Eulerian method to calculate the collection efficiency on two and three dimensional bodies," in 41st Aerospace Sciences Meeting and Exhibit, Reno, NV, 2003AIAA-2003-1073.

[20] Y. Bourgault, Z. Boutanios, and W. G. Habashi, "Threedimensional eulerian approach to droplet impingement simulation using FENSAP-ICE, part 1: model, algorithm, and validation," Journal of Aircraft, vol. 37, no. 1, pp. 95-103, 2000.

[21] X. Veillard, W. G. Habashi, M. S. Aubé et al., "FENSAP-ICE: ice accretion in multi-stage jet engines," in 19th AIAA Computational Fluid Dynamics, San Antonio, Texas, 2009AIAA2009-4158.

[22] C. N. Aliaga, M. S. Aubé, G. S. Baruzzi, and W. G. Habashi, "ENSAP-ICE-unsteady: unified in-flight icing simulation methodology for aircraft, rotorcraft, and jet engines," Journal of Aircraft, vol. 48, no. 1, pp. 119-126, 2011.

[23] Y. Bourgault, W. G. Habashi, J. Dompierre, and G. S. Baruzzi, "A finite element method study of Eulerian droplets impingement models," International Journal for Numerical Methods in Fluids, vol. 29, no. 4, pp. 429-449, 1999.

[24] E. Iuliano, V. Brandi, and G. Mingione, "Water impingement prediction on multi-element airfoils by means of eulerian and lagrangian approach with viscous and inviscid air flow," in 44th AIAA Aerospace Sciences Meeting and Exhibit, Reno, Nevada, 2006AIAA-2006-1270.

[25] C. Wang, S. Chang, M. Leng, B. Yang, and H. Wu, “A twodimensional splashing model for investigating impingement characteristics of supercooled large droplets," International Journal of Multiphase Flow, vol. 80, pp. 131-149, 2016.

[26] Ansys Inc, Ansys FLUENT User's Guide, Software Package, Ver. 19.1, ANSYS, Inc, Canonsburg, 2019.
[27] X. Shen, G. Lin, S. Yang, and H. Wang, "Eulerian method for droplets impingement calculation," in Proceedings of 48th SAFE Symposium, San Diego, CA, 2010.

[28] S. Yang, G. Lin, and X. Shen, "An Eulerian method for water droplet impingement prediction and its implementations," in Proceedings of the 1st International Symposium on Aircraft Airworthiness, pp. 72-81, Beijing, China, 2009. 